\title{
Effects of a Tumor Necrosis Factor- $\alpha$ Antagonist on Experimentally Induced Rhinosinusitis
}

\author{
Dong-Hyun Kim,, ${ }^{1}$ Eun-ju Jeon, ${ }^{1}$ Shi-Nae Park, ${ }^{2}$ Kyung-Ho Park, ${ }^{2}$ Yong-Soo Park, ${ }^{1}$ \\ and Sang Won Yeo ${ }^{2}$
}

\footnotetext{
${ }^{1}$ Department of Otolaryngology-Head and Neck Surgery, Incheon St. Mary's Hospital, College of Medicine, The Catholic University of Korea, 505 Banpo-Dong, Seocho-Gu, Seoul 137-040, Republic of Korea

${ }^{2}$ Department of Otolaryngology-Head and Neck Surgery, Seoul St. Mary's Hospital, College of Medicine, The Catholic University of Korea, 505 Banpo-Dong, Seocho-Gu, Seoul 137-040, Republic of Korea
}

Correspondence should be addressed to Sang Won Yeo, swyeo@catholic.ac.kr

Received 10 September 2010; Revised 14 March 2011; Accepted 3 April 2011

Academic Editor: Oreste Gualillo

\begin{abstract}
Copyright (C) 2011 Dong-Hyun Kim et al. This is an open access article distributed under the Creative Commons Attribution License, which permits unrestricted use, distribution, and reproduction in any medium, provided the original work is properly cited.
\end{abstract}

This prospective, randomized, and controlled study examined the effects of tumor necrosis factor soluble receptor type I (sTNFRI, a TNF- $\alpha$ antagonist) on experimentally induced rhinosinusitis in rats. The experimental groups received an instillation of lipopolysaccharide (LPS) plus an intramuscular injection of amoxicillin/clavulanate (antibiotic group), an instillation of sTNFRI (sTNFRI group), an instillation of sTNFRI and an injection of amoxicillin/clavulanate (sTNFRI/antibiotic group), or no additional treatment (LPS group). Histopathological changes were determined using hematoxylin-eosin and periodic acid-Schiff (PAS) staining. Leakage of exudate was determined using fluorescence microscopy. Vascular permeability was measured using the Evans blue dye technique. Expression of MUC5AC was measured using reverse transcriptase PCR. The sTNFRI, antibiotic, and sTNFRI/antibiotic groups had significantly less capillary permeability, mucosal edema, PAS staining, and expression of MUC5AC than the LPS group. There were no differences in capillary permeability, mucosal edema, PAS staining, and MUC5AC expression between the sTNFRI and sTNFRI/antibiotic groups. The antibiotic group had PAS staining similar to that of the sTNFRI and sTNFRI/antibiotic groups but had a greater increase in capillary permeability, mucosal edema, and MUC5AC expression. This study shows that sTNFRI reduces inflammatory activity and mucus hypersecretion in LPS-induced rhinosinusitis in rats.

\section{Introduction}

The respiratory tract is exposed to many external stimuli, among which are noxious gases, air pollutants, bacteria, and viruses. Exposure to harmful agents and microbial endotoxins can cause airway inflammations such as rhinosinusitis, inflammation of the mucosa of the nose and paranasal sinus [1]. Despite the development of new antibiotics and advances in sinus surgery over the past few decades, rhinosinusitis is still an enigmatic process. Moreover, treatment of rhinosinusitis with inappropriate antibiotics has contributed to the worldwide emergence of antibiotic-resistant strains of bacteria.

The recent appreciation that exposure to noninfectious inflammatory agents may predispose a person to infectious rhinosinusitis has stimulated renewed interest in the role of inflammatory mediators and inflammatory cells in the pathogenesis of rhinosinusitis [2, 3]. Many trials have highlighted the potential of inflammatory mediators for the treatment of inflammatory diseases, including rhinosinusitis [4-8].

Tumor necrosis factor (TNF) is an important mediator of inflammation and is produced by macrophages in response to stimuli such as bacterial lipopolysaccharide (LPS) and viruses [9]. It has been demonstrated that TNF- $\alpha$ antagonists block the activity of TNF and inhibit its action in vivo [68]. However, few studies have been conducted on the role of TNF- $\alpha$ antagonists in the treatment and prevention of rhinosinusitis. Therefore, this study examined the effects of a TNF- $\alpha$ antagonist on the LPS-induced inflammatory response in the nasal cavity and sinus of rats. 
TABLE 1: Histopathological changes in nasal mucosa (mean \pm SD).

\begin{tabular}{|c|c|c|c|c|c|c|}
\hline \multirow{2}{*}{ Group } & \multicolumn{2}{|c|}{ Absorbance } & \multicolumn{2}{|c|}{ Mucosal thickness (mm) } & \multicolumn{2}{|c|}{ PAS-stained area (\%) } \\
\hline & 1st day & 4th day & 1st day & 4th day & 1st day & 4th day \\
\hline Saline & $0.09 \pm 0.04$ & $0.10 \pm 0.02$ & $1.01 \pm 0.26$ & $1.21 \pm 0.67$ & $16.54 \pm 1.93$ & $16.39 \pm 1.34$ \\
\hline LPS & $0.17 \pm 0.03$ & $0.21 \pm 0.01$ & $2.11 \pm 1.27$ & $2.26 \pm 1.27$ & $22.24 \pm 2.90$ & $24.78 \pm 3.61$ \\
\hline LPS + sTNFRI & $0.12 \pm 0.01$ & $0.12 \pm 0.02$ & $1.32 \pm 0.66$ & $1.22 \pm 0.45$ & $15.81 \pm 1.64$ & $16.24 \pm 3.75$ \\
\hline LPS + antibiotic & $0.12 \pm 0.01$ & $0.19 \pm 0.01$ & $1.52 \pm 0.79$ & $1.97 \pm 0.93$ & $14.60 \pm 2.75$ & $16.82 \pm 1.06$ \\
\hline LPS + sTNFRI + antibiotic & $0.11 \pm 0.02$ & $0.11 \pm 0.01$ & $1.17 \pm 0.79$ & $1.31 \pm 0.57$ & $16.39 \pm 1.34$ & $16.31 \pm 4.81$ \\
\hline
\end{tabular}

TAвLE 2: Significance of differences ( $P$ value) in histopathological variables according to the time of sacrifice.

\begin{tabular}{lccc}
\hline & $\begin{array}{c}\text { Absorbance } \\
\text { 1st day versus 4th day }\end{array}$ & $\begin{array}{c}\text { Mucosal thickness (mm) } \\
\text { 1st day versus 4th day }\end{array}$ & $\begin{array}{c}\text { PAS-stained area (\%) } \\
\text { 1st day versus 4th day }\end{array}$ \\
\hline Saline & 0.467 & 0.427 & 0.196 \\
LPS & 0.333 & 0.803 & 0.099 \\
LPS + sTNFRI & 0.617 & 0.644 & 0.149 \\
LPS + antibiotic & $0.001^{*}$ & 0.189 & 0.863 \\
LPS + sTNFRI + antibiotic & 0.982 & 0.626 & 0.980 \\
\hline
\end{tabular}

${ }^{*}$ Significant $(P<.05)$.

\section{Materials and Methods}

2.1. Materials. The LPS used in this study was derived from Pseudomonas aeruginosa (L-4524, Sigma, St. Louis, Mo, USA). It was dissolved in normal saline solution at a concentration of $1 \mathrm{mg} / \mathrm{mL}$. TNF soluble receptor type I (sTNFRI) (PHR3015, Invitrogen, Camarillo, Calif, USA), a TNF- $\alpha$ antagonist was dissolved in a phosphate-buffered saline (0.1 M, pH 7.4) solution at a concentration of $0.2 \mathrm{mg} / \mathrm{mL}$.

Sixty-three healthy Sprague Dawley rats, weighing 200$250 \mathrm{~g}$ and free of pathogens and respiratory diseases according to the health and pathology reports of the supplier, were used in this study. All animals were housed and treated according to the regulations of the Catholic Ethics Committee of the Catholic University of Korea, which conformed to the NIH guidelines for the use of animals in research.

2.2. Methods. All experiments were performed with the rats subjected to $2 \%$ xylazine $(8 \mathrm{mg} / \mathrm{kg})$ anesthesia. Inhalant anesthesia was avoided to prevent irritation of the nasal mucosa. Both airways of the nasal cavity received an instillation of $0.1 \mathrm{~mL}$ of saline containing $0.1 \mathrm{mg}$ LPS once per day for 3 days. The instillate was deposited as a bead of fluid on the external nares, and the rats were allowed to aspirate it. Some rats were instilled with saline as a control. We carefully monitored breathing rate and skin color during instillation to prevent respiratory failure.

Sixty-three rats were allocated randomly to four treatment groups of 12 animals, each with 15 animals allocated to the control groups. One control group received no LPS or saline instillation (normal group; three animals), and the other control group was instilled with $0.1 \mathrm{~mL}$ of normal saline once per day for $3 \mathrm{~d}$ (saline group; 12 animals). All experimental groups received an instillation of LPS $(0.1 \mathrm{~mL})$ once per day for $3 \mathrm{~d}$. The LPS group received an LPS instillation alone, the sTNFRI group received an instillation of $0.1 \mathrm{~mL}$ of an sTNFRI solution, the antibiotic group received an intramuscular injection of $50 \mathrm{mg} / \mathrm{kg}$ amoxicillin/clavulanate, and the sTNFRI/antibiotic group received an intramuscular injection of $50 \mathrm{mg} / \mathrm{kg}$ amoxicillin/clavulanate and an instillation of $0.1 \mathrm{~mL}$ of an sTNFRI solution.

Each group except the normal group was subclassified into two subgroups based on the time of sacrifice (on the 1 st or 4th days after the final instillation of saline or LPS). Evans blue dye (E2129-10G, Sigma) was injected into the femoral vein at $20 \mathrm{mg} / \mathrm{mL}$ per kilogram of body weight 30$60 \mathrm{~min}$ before death. The rats turned blue immediately after infusion of the dye, confirming its uptake and distribution throughout the body.

The rats were exsanguinated $30 \mathrm{~min}$ after injection of dye and residual blood cells were flushed from the vascular system by perfusion of $100 \mathrm{~mL}$ of normal saline solution through an intra-aortic catheter. The nasal cavity was then lavaged with $0.1 \mathrm{~mL}$ of formamide for $5 \mathrm{~min}$ to collect the extravasated Evans blue dye.

After collecting the extravasated Evans blue dye, the head was removed and cleaned of skin and fur. A coronal incision was then made $1 \mathrm{~mm}$ posterior to the eyes to extract the maxilla (including the sinonasal cavity) for tissue processing. Half of the harvested bone was used for reverse transcriptase PCR analysis and half was used for staining. For staining, the bone was fixed in 10\% paraformaldehyde for $24 \mathrm{~h}$, decalcified in a rapid decalcifying solution (CalciClear Rapid, National Diagnostics, Atlanta, Ga, USA), embedded in a paraffin block, and cut into $4-5 \mu \mathrm{m}$ thick sections perpendicular to the plane of the hard palate. The mucosa of the maxillary sinus and nasal cavity was stained with periodic acid-Schiff (PAS) reagent and hematoxylin-eosin to determine histopathological changes.

2.3. Interpretation. The degree and location of the Evans blue dye extravasations in the nasal cavity and sinus 
TABLE 3: Significance of differences ( $P$ value) in histopathological variables between the LPS and other groups.

\begin{tabular}{lcccccc}
\hline & \multicolumn{2}{c}{ Absorbance } & \multicolumn{2}{c}{ Mucosal thickness (mm) } & \multicolumn{2}{c}{ PAS-stained area (\%) } \\
& 1 1st day & 4th day & 1st day & 4th day & 1 t day & 4th day \\
\hline LPS versus saline & $0.033^{*}$ & $0.031^{*}$ & 0.064 & $0.019^{*}$ & $0.001^{*}$ & $0.001^{*}$ \\
LPS versus LPS + sTNFRI & $0.001^{*}$ & $0.049^{*}$ & 0.171 & $0.011^{*}$ & $0.001^{*}$ & $0.001^{*}$ \\
LPS versus LPS + antibiotic & $0.002^{*}$ & 0.603 & 0.299 & 0.461 & $0.001^{*}$ & $0.006^{*}$ \\
LPS versus LPS + sTNFRI + antibiotic & $0.001^{*}$ & $0.046^{*}$ & 0.116 & $0.014^{*}$ & $0.001^{*}$ & $0.008^{*}$ \\
\hline
\end{tabular}

${ }^{*}$ Significant $(P<.05)$.

TABLE 4: Significance of differences ( $P$ value) in histopathological variables between treatments.

\begin{tabular}{lcccccc}
\hline & \multicolumn{2}{c}{ Absorbance } & \multicolumn{2}{c}{ Mucosal thickness (mm) } & \multicolumn{2}{c}{ PAS-stained area (\%) } \\
& 1st day & 4th day & 1st day & 4th day & 1st day & 4th day \\
\hline sTNFRI versus antibiotic & 0.191 & $0.001^{*}$ & 0.519 & $0.008^{*}$ & 0.287 & 0.819 \\
Antibiotic versus sTNFRI + antibiotic & 0.096 & $0.001^{*}$ & 0.305 & $0.022^{*}$ & 0.197 & 0.873 \\
sTNFRI versus sTNFRI + antibiotic & 0.489 & 0.743 & 0.624 & 0.659 & 0.528 & 0.987 \\
\hline
\end{tabular}

${ }^{*}$ Significant $(P<.05)$.

mucosa were examined on unstained slides using confocal scanning microscopy ( $543 \mathrm{~nm}$, Bio-Rad Radiance Plus, BioRad, Hemel Hempstead, UK). To quantify the amount of extravasated dye, absorbance of the supernatant at $630 \mathrm{~nm}$ was measured using a spectrophotometer (Du-530, Beckman Coulter, Fullerton, Calif, USA).

The thickness of the mucosa was defined as the maximum thickness of the mucosa overlying the maxillary sinus and was measured at a magnification of $\times 400$. The mean mucosal thickness was calculated using three sections per group.

MetaMorph imaging software (Molecular Devices Inc., Downing Town, Pa, USA) was used to evaluate the area of PAS staining [10-12] in the mucosa of the nasal opening at the base of the nasal septum. The total area of mucosa of the nasal opening at the base of the nasal septum was also measured, and the PAS-stained area was expressed as a percentage of the total area. Image analysis was performed using a blinded protocol.

The other half of the harvested bone was homogenized, frozen in liquid nitrogen, and stored at $-70^{\circ} \mathrm{C}$. RNA was then extracted using an extraction kit (iNtRON Biotechnology, Inc., Gyeonggi-do, Korea) according to the manufacturer's instructions.

Polymerase activation for MUC5AC was performed at $95^{\circ} \mathrm{C}$ for $15 \mathrm{~min}$ followed by 32 cycles at $94^{\circ} \mathrm{C}$ for $1 \mathrm{~min}, 55^{\circ} \mathrm{C}$ for $1 \mathrm{~min}$, and $72^{\circ} \mathrm{C}$ for $1 \mathrm{~min}$. Polymerase activation for glyceraldehyde 3-phosphate dehydrogenase (GAPDH) was performed at $94^{\circ} \mathrm{C}$ for $4 \mathrm{~min}$, followed by 35 cycles at $94^{\circ} \mathrm{C}$ for $1 \mathrm{~min}, 60^{\circ} \mathrm{C}$ for $1 \mathrm{~min}$, and $72^{\circ} \mathrm{C}$ for $1 \mathrm{~min}$.

The primer sequences were obtained from GenBank and were designed using Gene Runner software (Hastings Software Inc., Las Vegas, Nev, USA). The sequences of the primers were as follows: MUC5AC: forward, $5^{\prime}$-CATAGCCTCCTCTTGTTC- $3^{\prime}$ and reverse, $3^{\prime}$-ATTCCTGTAGCAGTAGTGAG-5'; and GAPDH: forward, $5^{\prime}$-GCTGGTGCTGAGTATGTCGT- $3^{\prime}$ and reverse, $3^{\prime}$-GAATGGGAGTTGCTGTTGAA-5'. GAPDH was used as a constitutive control.
The products were separated by agarose gel electrophoresis and visualized using ethidium bromide staining. The bands were digitized using a Bio-Rad Universal Hood system (BioRad, Hercules, Calif, USA). The mean MUC5AC to GAPDH band photodensity ratio was calculated for each group.

2.4. Statistics. Group means were compared using the Kruskal-Wallis test and SAS version 8.1 software (SAS institute, Inc., Cary, NC, USA). The results are presented as the mean \pm standard deviation. A $P$ value $<.05$ was considered significant.

\section{Results and Discussion}

3.1. Changes in the Microvascular Permeability of the Mucosa of the Nasal Cavity and Sinus. The mean absorbance of the LPS group increased with time, but the increase was not significant. The mean absorbances of all other groups except the antibiotic group showed no significant changes with time. The antibiotic group showed a significant increase in absorbance with time (Tables 1 and 2). The mean absorbance of the LPS group was significantly greater than that of the saline group on the 1st and 4th days (Tables 1 and 3 ).

The mean absorbances of the LPS group were compared with those of the sTNFRI, antibiotic, and sTNFRI/antibiotic groups. Although there was no significant difference between the LPS group and the antibiotic group on the 4th day, the mean absorbances of the other study groups were significantly less than those of the LPS groups (Tables 1 and 3).

Mean absorbances were compared between the sTNFRI, antibiotic, and sTNFRI/antibiotic groups. There was no significant difference in absorbance between groups except for that of the antibiotic subgroup that was sacrificed on the 4th day, which was significantly greater than those of the sTNFRI and sTNFRI/antibiotic groups on the 4th day (Tables 1 and 4). 


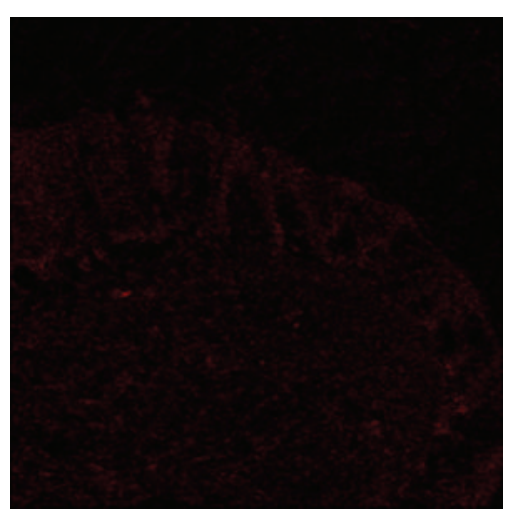

(a)

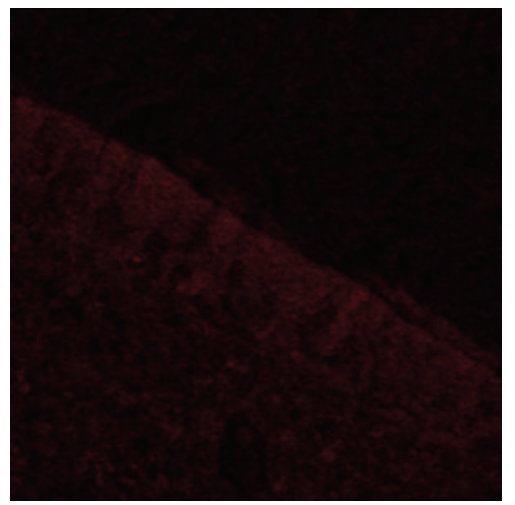

(d)

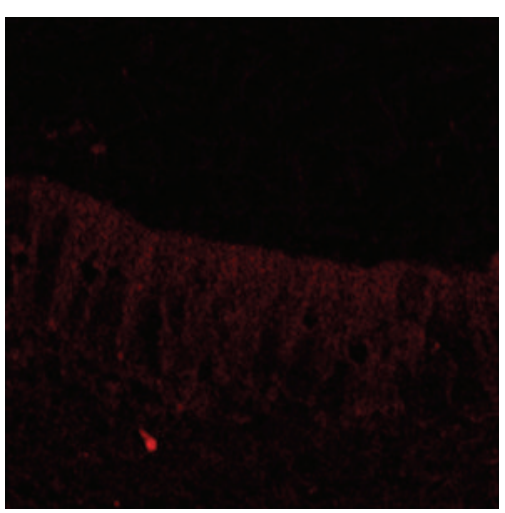

(b)

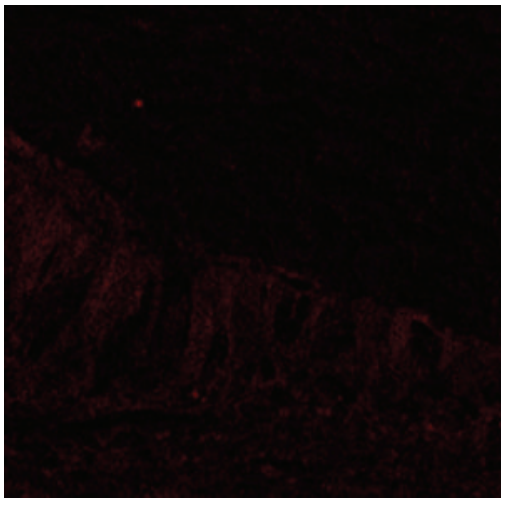

(e)

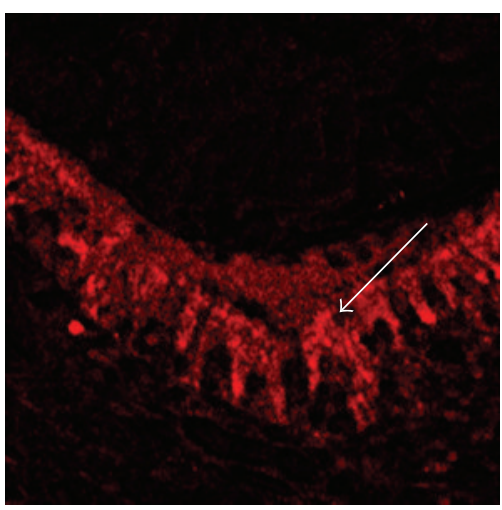

(c)

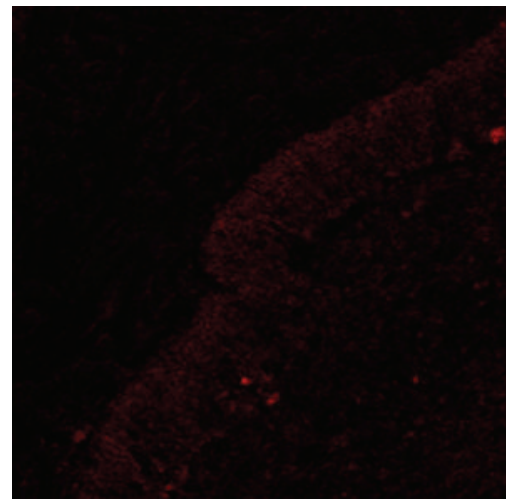

(f)

FIGURE 1: Fluorescence microscope images of Evans blue dye leakage on the 4th day. (a) Normal group; (b) saline group; (c) LPS group; (d) sTNFRI group; (e) antibiotic group; (f) sTNFRI/antibiotic group. Massive leakage of Evans blue dye was observed in the mucosa of the LPS group ((c), arrows) $(\times 400)$.

Little Evans blue dye was extravasated in the saline, sTNFRI, antibiotic, and sTNFRI/antibiotic groups. However, Evans blue dye was extravasated into the mucosa in the LPS group (Figure 1).

3.2. Thickness of the Maxillary Sinus Mucosa. There were no significant differences in mucosal thickness between the 1st day and the 4th day. The mean mucosal thickness of the LPS group, antibiotic group, and sTNFRI/antibiotic group increased with time, whereas the mean mucosal thickness of the sTNFRI group tended to decrease with time (Tables 1 and 2).

Compared with the saline group, the mean mucosal thickness of the LPS group increased significantly on the 4th day (Tables 1 and 3). The mean mucosal thickness of the LPS group was compared with those of the sTNFRI, antibiotic, and sTNFRI/antibiotic groups. Although the mean mucosal thickness of the sTNFRI, antibiotic, and sTNFRI/antibiotic groups was less than that of the LPS group on the 1st day, there was no significant difference between each group and the LPS group on the 1st day. The mean mucosal thicknesses of the sTNFRI and sTNFRI/antibiotic groups were significantly less than that of the LPS group on the 4 th day (Figure 2). Although the mean mucosal thickness of the antibiotic group appeared to be less than that of the
LPS group on the 4th day, the difference was not statistically significant (Tables 1 and 3 ).

Mean mucosal thickness was compared between the sTNFRI, antibiotic, and sTNFRI/antibiotic groups. There were no significant differences between the three groups on the 1st day. However, there were significant differences between the sTNFRI and antibiotic groups, as well as between the antibiotic and sTNFRI/antibiotic groups, on the 4th day. There was no significant difference between the sTNFRI and sTNFRI/antibiotic groups on the 4 th day (Tables 1 and 4).

3.3. Morphometry. The mean area of PAS staining of the LPS group increased with time, but the increase was not significant. The mean areas of PAS staining of the other groups did not change significantly with time (Tables 1 and 2).

Compared with the saline group, the PAS-stained areas of the LPS group were significantly elevated on the 1st and 4 th days (Tables 1 and 3 ). The mean areas of PAS staining of the LPS groups were compared with those of the sTNFRI, antibiotic, and sTNFRI/antibiotic groups (Figure 3 ). The mean areas of PAS staining of all study groups were significantly lower than those of the LPS groups on the 1st and 4 th days (Tables 1 and 3 ). The mean areas of PAS staining 


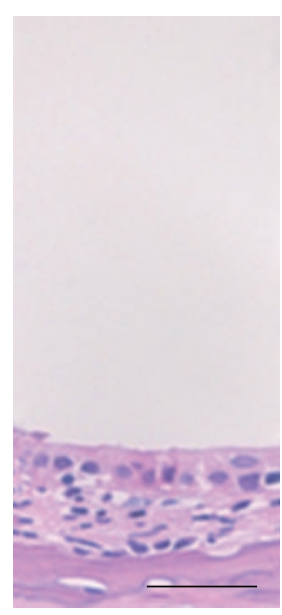

(a)

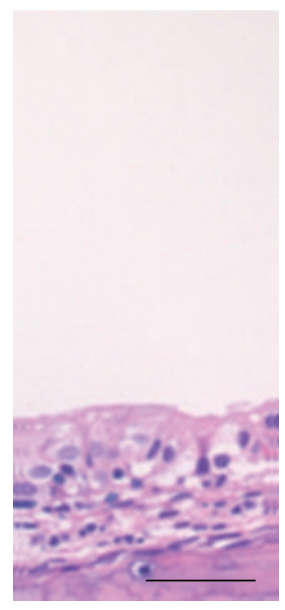

(d)

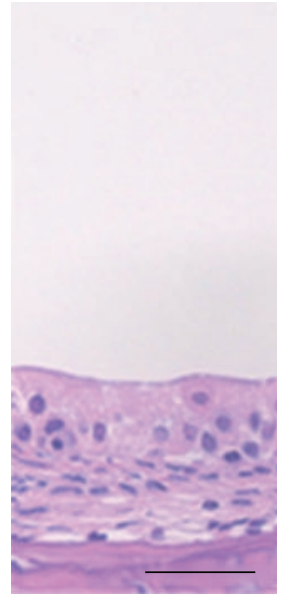

(b)

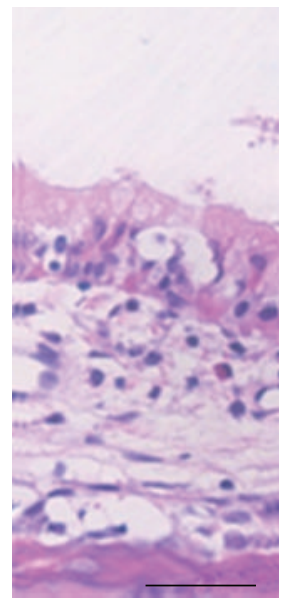

(e)

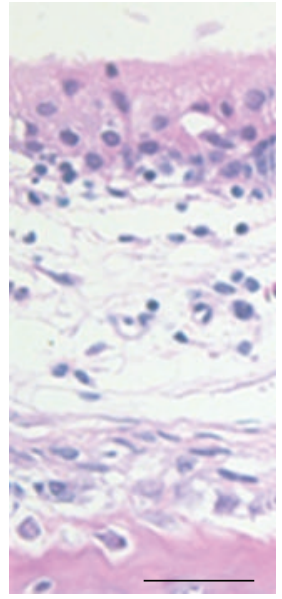

(c)

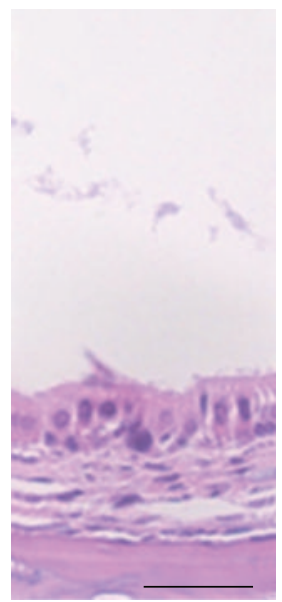

(f)

Figure 2: Photomicrographs of sinus mucosa on the 4th day. The mucosa was thin in the normal (a) and saline (b) control groups. In the LPS group (c), the mucosa was markedly thickened. The sTNFRI (d), antibiotic (e), and sTNFRI/antibiotic (f) groups showed significant decreases in mucosal thickening compared with the LPS group (c) (hematoxylin-eosin staining; scale bar $=1 \mathrm{~mm}$ ).

of the sTNFRI, antibiotic, and sTNFRI/antibiotic groups were similar on the 1st and 4th days (Tables 1 and 4 ).

3.4. MUC5AC Expression in the Nasal Cavity and Sinus. Compared with the saline control group, LPS induced a 10.5 -fold increase in mucin gene expression on the 1st day (Figure 4) and a 6.5-fold increase in mucin gene expression on the 4th day (Figure 5). Upregulation of MUC5AC mRNA expression was significantly inhibited in the sTNFRI, antibiotic, and sTNFRI/antibiotic groups. The level of MUC5AC mRNA expression in the antibiotic group was significantly higher than that in the sTNFRI and sTNFRI/antibiotic groups on the 1 st and 4 th days $(P=.001)$.

This study examined inflammatory responses in rats with LPS-induced rhinosinusitis. LPS was used as an infectious agent instead of a bacterium. Streptococcus pneumoniae, Haemophilus influenza, and Moraxella catarrhalis are cited as the most common bacterial pathogens responsible for acute sinusitis [13]. Gram-negative bacteria such as Klebsiella pneumoniae, Pseudomonas aeruginosa, Serratia marcescens, and Proteus mirabilis are reported to cause sinusitis infrequently, mainly in immunocompromised patients [14]. Recent investigations indicated that Gram-negative bacteria are frequently involved in sinusitis that is recalcitrant to traditional medical and surgical therapy, even in immunocompetent patients $[15,16]$. LPS is the primary component of endotoxin, a mixture of Gram-negative bacteria cell wall components [17]. Endotoxin is not readily eradicated by the local host defense mechanism and has been shown to persist for up to three months, even after effective antibiotic treatment $[6,18]$.

In the present study, capillary permeability, mucosal edema, PAS staining, and expression of MUC5AC were examined on the 1st and 4th days after the final instillation of LPS. The percentage area stained by PAS, which indicates the production of mucosubstance and the mRNA expression of MUC5AC, a major mucin secreted from the goblet cells of the surface epithelium and the most important mucin in the pathogenesis of mucus hypersecretion [19-21], were analyzed to evaluate mucus hypersecretion. 


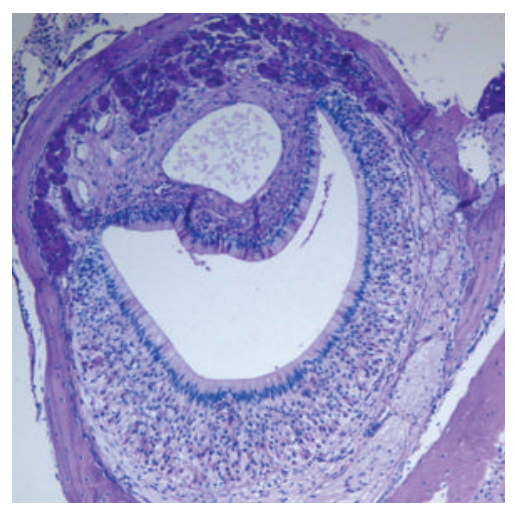

(a)

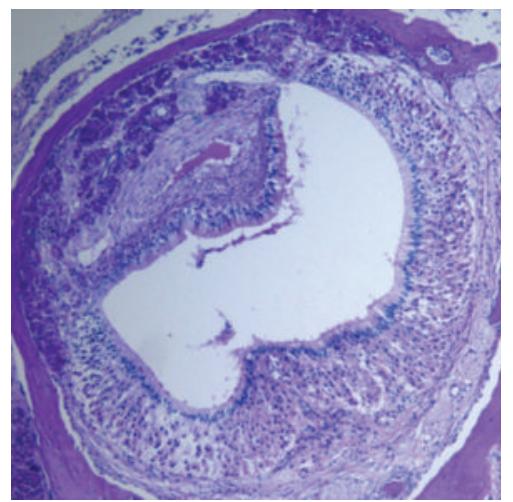

(d)

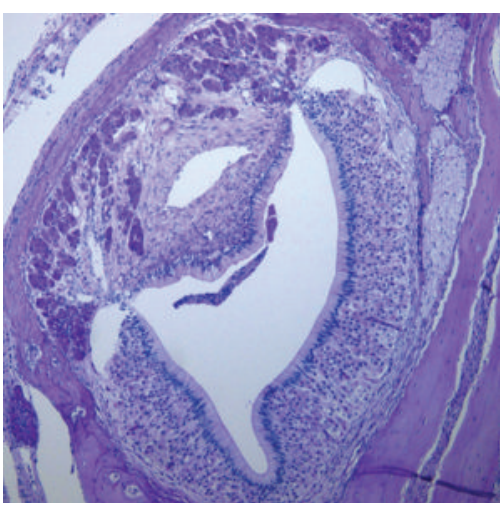

(b)

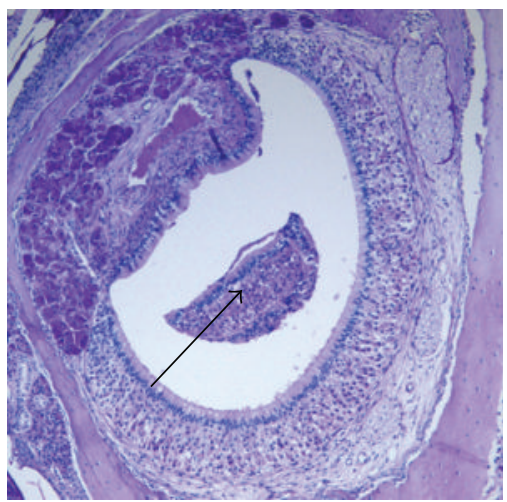

(e)

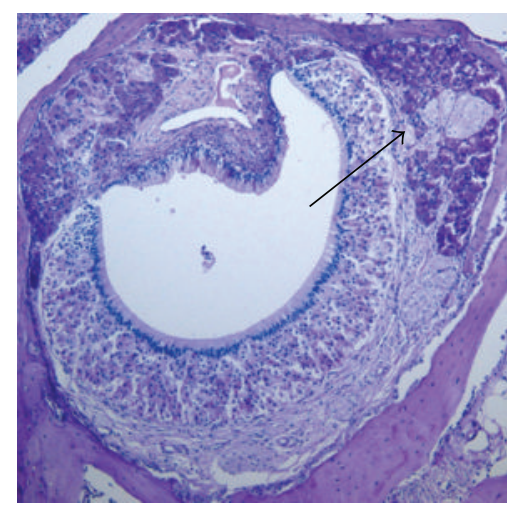

(c)

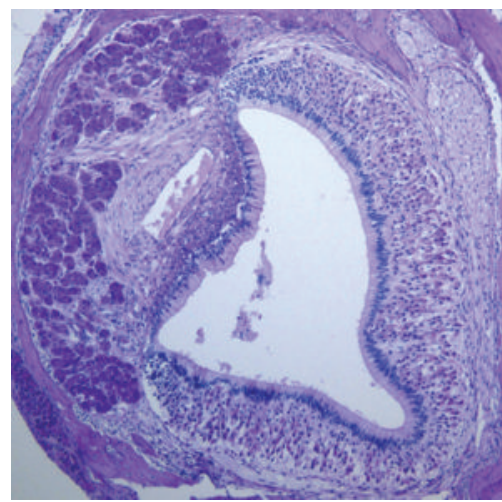

(f)

FIgURE 3: Histopathological images of nose-tissue sections stained with PAS on the 4th day. (a) Normal group; (b) saline group; (c) LPS group; (d) sTNFRI group; (e) antibiotic group; (f) sTNFRI/antibiotic group. A large PAS-stained area was observed in the LPS group ((c), arrow), and luminal inflammatory cell clumping was noted in the antibiotic group $((\mathrm{e})$, arrow $)(\times 200)$.

Compared with intranasal saline instillation, intranasal LPS instillation significantly increased microvascular permeability, mucosal thickness, and the percentage of PAS-stained area and induced overexpression of MUC5AC on the 1st and 4th days. Inflammation and mucus hypersecretion of the sinonasal tract were observed after LPS instillation. These findings are similar to the characteristics of the rat model of platelet activating factor-induced rhinosinusitis [22].

Although the mean absorbance, mucosal thickness, and PAS-stained area of the LPS groups increased with time, the increases were not significant. LPS induced a 10.5-fold increase in mucin gene expression compared with the saline control group on the 1st day and a 6.5 -fold increase in mucin gene expression on the 4 th day.

We did not document the time course of inflammation in this study. Liu et al. reported that the threshold time point for inducible nitric oxide synthase mRNA induction was between $20 \mathrm{~min}$ and $40 \mathrm{~min}$ after LPS administration, and that the quantity of mRNA increased progressively thereafter, reaching a plateau between $4 \mathrm{~h}$ and $8 \mathrm{~h}$, and decreasing markedly by $24 \mathrm{~h}$ in rats treated with LPS [23]. We consider the time course reported by Liu et al. applicable to our study. Further studies are required to determine the exact time course of inflammation after intranasal LPS instillation. To verify our hypothesis, additional studies on the relationship between inducible nitric oxide synthase messenger RNA and LPS-induced rhinosinusitis are needed.

Antibiotics are commonly used to treat bacterial rhinosinusitis. Amoxicillin is generally used as a first treatment, and amoxicillin/clavulanate is indicated when the patient's symptoms do not improve with amoxicillin alone [17].

Although the LPS-induced expression of MUC5AC and the increase in PAS staining were significantly reduced by amoxicillin/clavulanate compared with the LPS group, LPS-induced increases in microvascular permeability and mucosal thickness were less affected. In addition, LPSstimulated expression of MUC5AC was higher in the antibiotic group than that in the sTNFRI group. The antibiotic group had greater microvascular permeability, mucosal thickness, and MUC5AC mRNA expression than the sTNFRI groups. This difference was significant on the 4th day. These results show that amoxicillin/clavulanate is less effective in treating LPS-induced rhinosinusitis than sTNFRI. Because the agent used to induce rhinosinusitis in this study was not a bacterium but LPS, it appears that amoxicillin/clavulanate, which acts by inhibiting the synthesis of bacterial cell walls, was less effective than sTNFRI in treating LPS-induced rhinosinusitis. Ou et al. reported that amoxicillin had no effect on MUC5AC expression during a pulmonary inflammatory response to LPS [24]. 


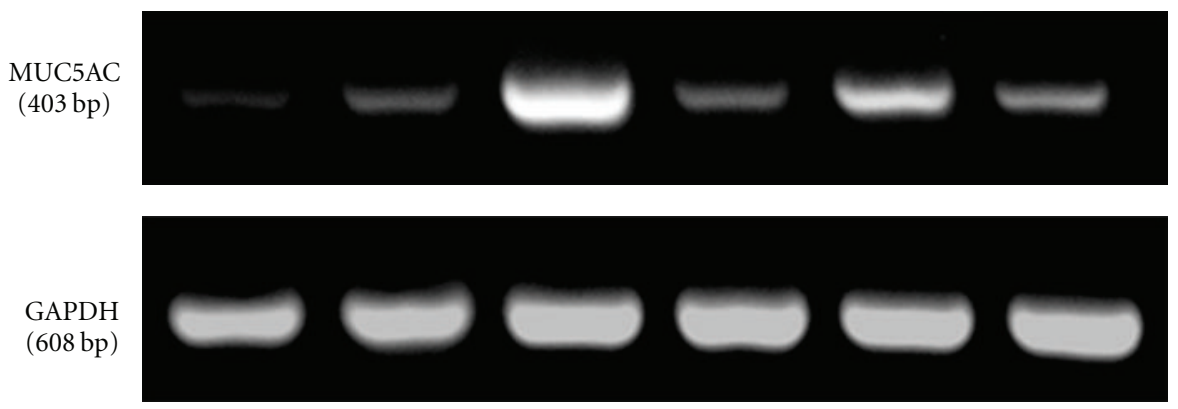

(a) Representative agarose gel showing the results of RT-PCR analysis for mRNA

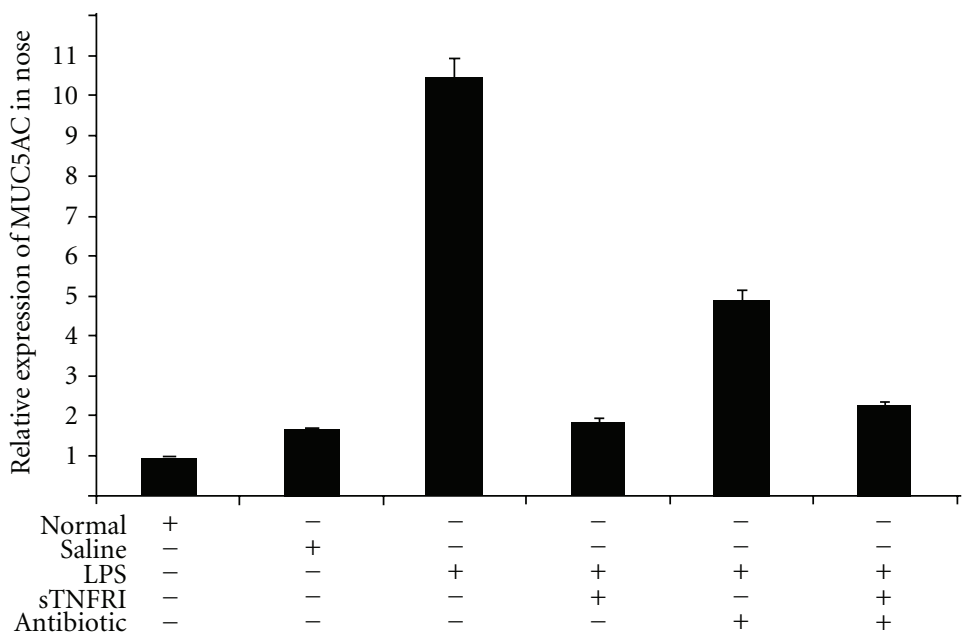

(b) Optical density ratio of MUC5AC mRNA as assessed by image analysis

FIgURE 4: Expression of MUC5AC mRNA in the rat nose on the 1st day after the last instillation of LPS. Expression of MUC5AC mRNA increased significantly in rats treated with LPS, and the upregulation of MUC5AC mRNA expression was inhibited by sTNFRI, antibiotic, and sTNFRI/antibiotic $(P<.05)$.

Cytokines such as interleukin (IL)-1 beta, IL-8, and TNF- $\alpha$ are produced by monocytes and play an important role in the inflammatory response to LPS [25-27]. The proinflammatory cytokines, IL- 1 beta, and TNF- $\alpha$ stimulate neutrophils to release oxidants and proteases and stimulate endothelial cells to produce vascular cell adhesion molecule1 (VCAM-1) and intercellular adhesion molecule-1 (ICAM1), which facilitate the adherence of leukocytes to vascular endothelial cells. Therefore, leukocytes infiltrate the airways and cause injury by releasing inflammatory products [2831]. Tumor necrosis factor-soluble receptor (sTNFR) acts as an inhibitor by binding to TNF- $\alpha$ and preventing its binding to the cell surface receptor. Hence, it inhibits the activity of TNF- $\alpha$. Two types of sTNFR are recognized: sTNFRI (60 kD) and sTNFRII $(80 \mathrm{kD})$. sTNFRI is more potent than sTNFRII [32]. Lee et al. reported that sTNFRI prevented experimental otitis media when effusion was induced using TNF- $\alpha$ [6]. Kim et al. demonstrated that sTNFRI prevents experimental otitis media when effusion is induced using LPS [7].

Based on these reports $[6,7,25-32]$, this study examined the preventive effects of a TNF- $\alpha$ antagonist on inflammatory responses induced by LPS in the nasal cavity and sinus. intranasal instillation of sTNFRI attenuated LPS-induced increases in microvascular permeability and PAS staining in the sinonasal tract on the 1st and 4th days. sTNFRI reduced LPS-induced increases in mucosal thickness on the 4th day. In addition, sTNFRI attenuated LPS-induced mRNA overexpression of MUC5AC on the 1st and 4th days. These attenuating effects were similar on the 1st and 4th days. These results suggest that sTNFRI suppresses both inflammation and mucus hypersecretion by downregulating mRNA expression of MUC5AC. Therefore, intranasal instillation of sTNFRI effectively prevents LPS-induced rhinosinusitis in rats.

The sTNFRI/antibiotic combination attenuated LPSinduced increases in microvascular permeability and PAS staining on the 1 st and 4 th days. The sTNFRI/antibiotic combination reduced LPS-induced increases in mucosal thickness on the 4th day and attenuated LPS-induced mRNA overexpression of MUC5AC on the 1st and 4th days.

As the effects of sTNFRI/antibiotic bear greater similarity to those of the sTNFRI group than those of the antibiotic group, the effects of the sTNFRI/antibiotic combination are probably due to the effect of the sTNFRI component rather than that of the antibiotic component. 


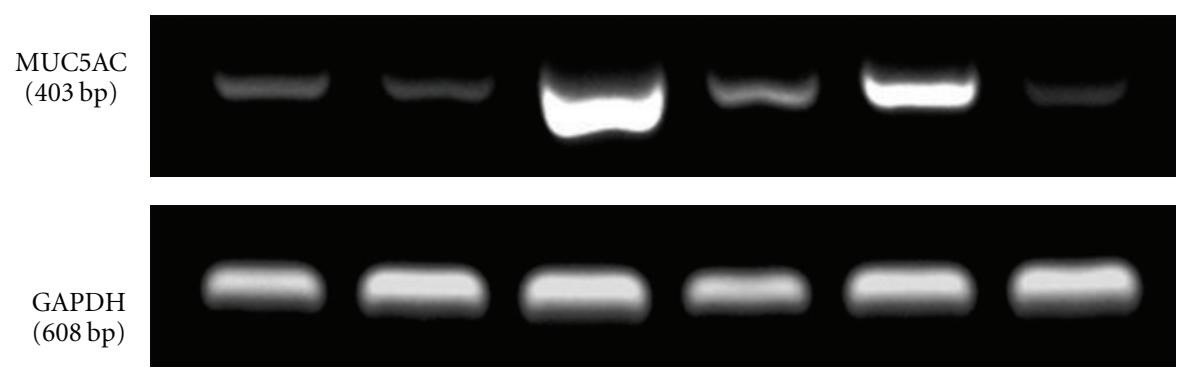

(a) Representative agarose gel showing the results of RT-PCR analysis for mRNA

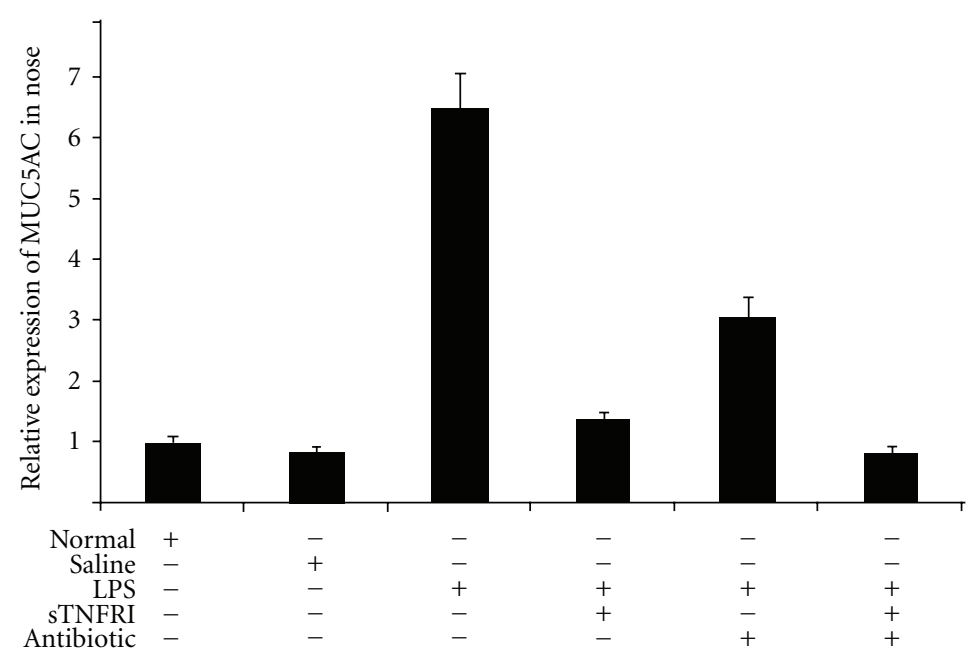

(b) Optical density ratio of MUC5AC mRNA as assessed by image analysis

FIgURE 5: Expression of MUC5AC mRNA in the rat nose on the 4th day after the final instillation of LPS. The level of MUC5AC mRNA expression was significantly higher in rats treated with LPS, and upregulation of MUC5AC mRNA expression was inhibited by sTNFRI, antibiotic, and sTNFRI/antibiotic $(P<.05)$.

Further studies to compare the effects of other inhibitors (such as a platelet-activating factor antagonist) with those of sTNFRI are needed to identify more potent inhibitors of LPSinduced rhinosinusitis.

\section{Conclusions}

Intranasal administration of sTNFRI reduces inflammatory activity, including elevated microvascular permeability and tissue edema, and mucus hypersecretion, including production of mucosubstance and expression of MUC5AC, in LPSinduced rhinosinusitis in rats. These results demonstrate that intranasal administration of sTNFRI reduces inflammatory activity and mucus hypersecretion in LPS-induced rhinosinusitis in rats.

\section{Acknowledgment}

This study was supported by the Incheon St. Mary's Hospital of the Catholic University of Korea through the Clinical Research Laboratory Foundation Program.

\section{References}

[1] R. Rylander, "Endotoxin in the environment-exposure and effects," Journal of Endotoxin Research, vol. 8, no. 4, pp. 241252, 2002.

[2] S. M. Mucha and F. M. Baroody, "Sinusitis update," Current Opinion in Allergy and Clinical Immunology, vol. 3, no. 1, pp. 33-38, 2003.

[3] E. Appenroth, A. R. Gunkel, H. Müller, C. Völklein, and A. Schrott-Fischer, "Activated and non-activated eosinophils in patients with chronic rhinosinusitis," Acta Oto-Laryngologica, vol. 118, no. 2, pp. 240-242, 1998.

[4] D. S. Liu, W. J. Liu, L. Chen et al., "Rosiglitazone, a peroxisome proliferator-activated receptor- $\gamma$ agonist, attenuates acroleininduced airway mucus hypersecretion in rats," Toxicology, vol. 260, no. 1-3, pp. 112-119, 2009.

[5] L. Chen, T. Wang, J. Y. Zhang et al., “Toll-like receptor 4 relates to lipopolysaccharide-induced mucus hypersecretion in rat airway," Archives of Medical Research, vol. 40, no. 1, pp. 10-17, 2009.

[6] D. H. Lee, Y. S. Park, T. T. K. Jung, S. W. Yeo, Y. C. Choi, and E. J. Jeon, "Effect of tumor necrosis factor- $\alpha$ on experimental otitis media with effusion," Laryngoscope, vol. 111, no. 4, part 1, pp. 728-733, 2001. 
[7] D. H. Kim, Y. S. Park, E. J. Jeon, S. W. Yeo, K. H. Chang, and S. K. Lee, "Effects of tumor necrosis factor alpha antagonist, platelet activating factor antagonist, and nitric oxide synthase inhibitor on experimental otitis media with effusion," The Annals of Otology, Rhinology and Laryngology, vol. 115, no. 8, pp. 617-623, 2006.

[8] D. H. Lee, S. W. Yeo, K. H. Chang, S. Y. Park, J. H. Oh, and J. H. Seo, "Effect of infliximab on experimentally induced otitis media in rats," The Annals of Otology, Rhinology and Laryngology, vol. 117, no. 6, pp. 470-476, 2008.

[9] D. Ophir, T. Hahn, A. Schattner, D. Wallach, and A. Aviel, "Tumor necrosis factor in middle ear effusions," Archives of Otolaryngology-Head \& Neck Surgery, vol. 114, no. 11, pp. 1256-1258, 1988.

[10] J. S. Francisco, H. P. Moraes, and E. P. Dias, "Evaluation of the Image-Pro Plus 4.5 software for automatic counting of labeled nuclei by PCNA immunohistochemistry," Brazilian Oral Research, vol. 18, no. 2, pp. 100-104, 2004.

[11] J. H. Kim, S. Y. Lee, S. M. Bak et al., "Effects of matrix metalloproteinase inhibitor on LPS-induced goblet cell metaplasia," American Journal of Physiology, vol. 287, no. 1, pp. L127-L133, 2004.

[12] T. Shimizu, H. Hirano, S. Shimizu, C. Kishioka, Y. Sakakura, and Y. Majima, "Differential properties of mucous glycoproteins in rat nasal epithelium: a comparison between allergic inflammation and lipopolysaccharide stimulation," The American Journal of Respiratory and Critical Care Medicine, vol. 164, no. 6, pp. 1077-1082, 2001.

[13] I. Brook, "Microbiology and antimicrobial management of sinusitis," Journal of Laryngology and Otology, vol. 119, no. 4, pp. 251-258, 2005.

[14] S. W. Cheung, K. C. Lee, and I. Cha, "Orbitocerebral complications of pseudomonas sinusitis," Laryngoscope, vol. 102, no. 12, part 1, pp. 1385-1389, 1992.

[15] W. E. Bolger, D. Leonard, E.J. Dick Jr., and P. Stierna, "Gramnegative sinusitis: a bacteriologic and histologic study in rabbits," American Journal of Rhinology, vol. 11, no. 11, pp. 1525, 1997.

[16] J. Hsu, D. C. Lanza, and D. W. Kennedy, "Antimicrobial resistance in bacterial chronic sinusitis," American Journal of Rhinology, vol. 12, no. 4, pp. 243-248, 1998.

[17] R. S. Leung and R. Katial, "The diagnosis and management of aute and chronic sinusitis," Primary Care, vol. 35, no. 1, pp. 11-24, 2008.

[18] L. P. Schousboe, T. Ovesen, and C. B. Pedersen, "Middle ear epithelium has inflammatory capacity," Acta Oto-Laryngologica, Supplement, no. 543, pp. 89-91, 2000.

[19] M. Z. Alimam, F. M. Piazza, D. M. Selby, N. Letwin, LI. Huang, and M. C. Rose, "Muc-5/5ac mucin messenger RNA and protein expression is a marker of goblet cell metaplasia in murine airways," American Journal of Respiratory Cell and Molecular Biology, vol. 22, no. 3, pp. 253-260, 2000.

[20] M. C. Rose, F. M. Piazza, Y. A. Chen et al., "Model systems for investigating mucin gene expression in airway diseases," Journal of Aerosol Medicine: Deposition, Clearance, and Effects in the Lung, vol. 13, no. 3, pp. 245-261, 2000.

[21] K. S. Song, W. J. Lee, K. C. chung et al., "Interleukin-1 $\beta$ and tumor necrosis factor- $\alpha$ induce MUC5AC overexpression through a mechanism involving ERK/p38 mitogen-activated protein kinases-MSK1-CREB activation in human airway epithelial cells," The Journal of Biological Chemistry, vol. 278, no. 26, pp. 23243-23250, 2003.

[22] S. Y. Jeon, J. P. Kim, S. K. Ahn, E. A. Kim, and B. G. Kim, "Rat model of platelet-activating factor-induced rhinosinusitis,"
The Annals of Otology, Rhinology and Laryngology, vol. 114, no. 5, pp. 393-398, 2005.

[23] S. F. Liu, P. J. Barnes, and T. W. Evans, "Time course and cellular localization of lipopolysaccharide-induced inducible nitric oxide synthase messenger RNA expression in the rat in vivo," Critical Care Medicine, vol. 25, no. 3, pp. 512-518, 1997.

[24] X.-M. Ou, Y.-L. Feng, F.-Q. Wen et al., "Macrolides attenuate mucus hypersecretion in rat airways through inactivation of NF- $\kappa$ B," Respirology, vol. 13, no. 1, pp. 63-72, 2008.

[25] G. C. Higgins, J. L. Foster, and A. E. Postlethwaite, "Interleukin $1 \beta$ propeptide is detected intracellularly and extracellularly when human monocytes are stimulated with LPS in vitro," The Journal of Experimental Medicine, vol. 180, no. 2, pp. 607-614, 1994.

[26] G. Lin, A. E. Pearson, R. W. Scamurra et al., "Regulation of interleukin-8 expression in porcine alveolar macrophages by bacterial lipopolysaccharide," The Journal of Biological Chemistry, vol. 269, no. 1, pp. 77-85, 1994.

[27] H. Ohtsuka, K. Ohki, T. Tanaka, M. Tajima, T.-O. Yoshino, and K. Takahashi, "Circulating tumor necrosis factor and interleukin-1 after administration of LPS in adult cows," Journal of Veterinary Medical Science, vol. 59, no. 10, pp. 927929, 1997.

[28] B. O. Anderson, J. M. Brown, D. D. Bensard et al., "Reversible lung neutrophil accumulation can cause lung injury by elastase-mediated mechanisms," Surgery, vol. 108, no. 2, pp. 262-268, 1990.

[29] M. Kishi, L. F. Richard, R. O. Webster, and T. E. Dahms, "Role of neutrophils in xanthine/xanthine oxidase-induced oxidant injury in isolated rabbit lungs," Journal of Applied Physiology, vol. 87, no. 6, pp. 2319-2325, 1999.

[30] K. Kawabata, T. Hagio, and S. Matsuoka, "The role of neutrophil elastase in acute lung injury," European Journal of Pharmacology, vol. 451, no. 1, pp. 1-10, 2002.

[31] B. C. Sheridan, R. C. McIntyre, E. E. Moore, D. R. Meldrum, J. Agrafojo, and D. A. Fullerton, "Neutrophils mediate pulmonary vasomotor dysfunction in endotoxin-induced acute lung injury," Journal of Trauma, vol. 42, no. 3, pp. 391-397, 1997.

[32] K. Maxwell, G. Leonard, and D. L. Kreutzer, "Cytokine expression in otitis media with effusion: tumor necrosis factor soluble receptor," Archives of Otolaryngology-Head \& Neck Surgery, vol. 123, no. 9, pp. 984-988, 1997. 


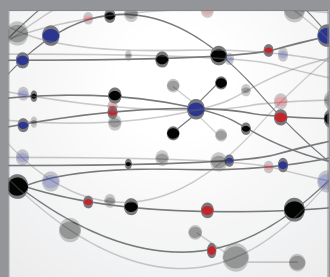

The Scientific World Journal
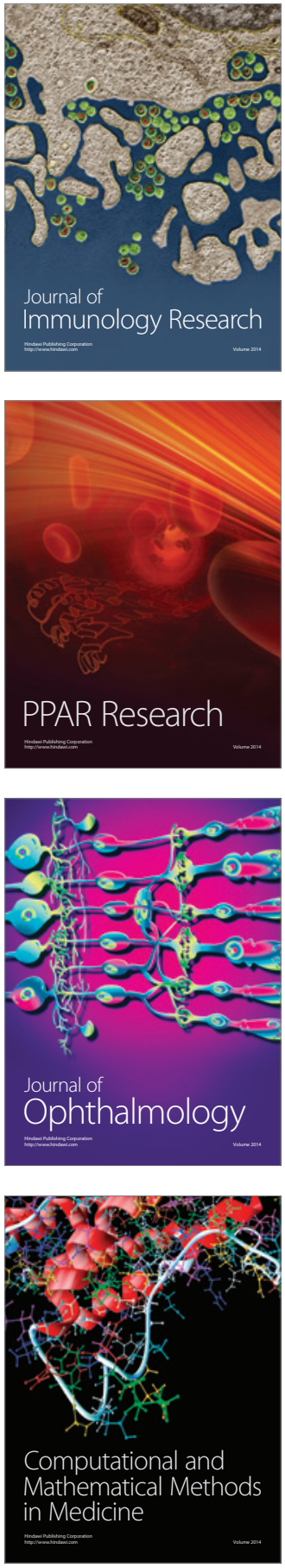

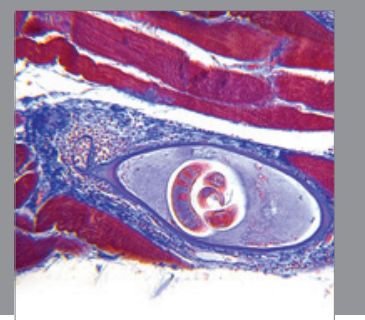

Gastroenterology

Research and Practice
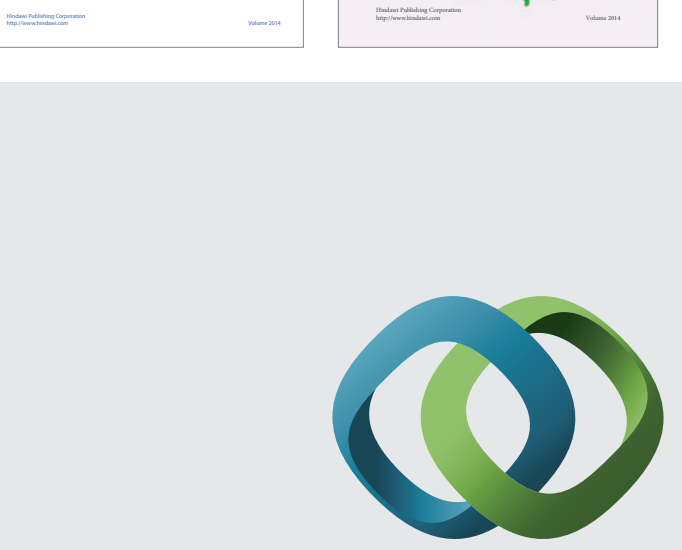

\section{Hindawi}

Submit your manuscripts at

http://www.hindawi.com
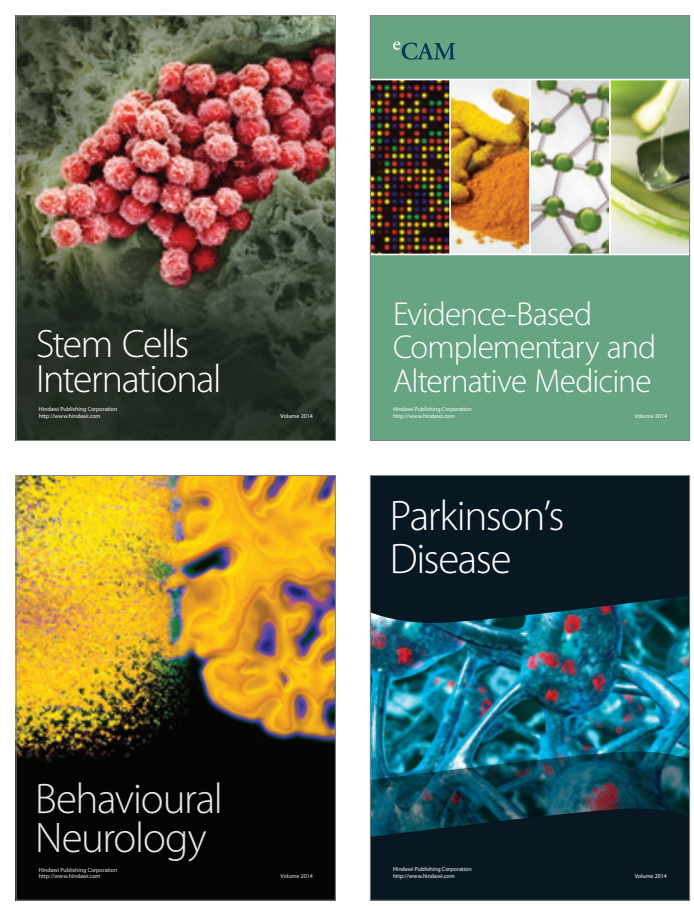

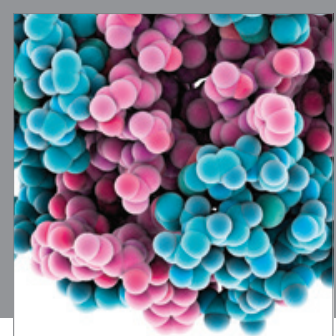

Journal of
Diabetes Research

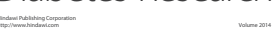

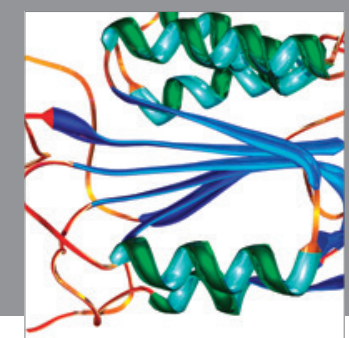

Disease Markers
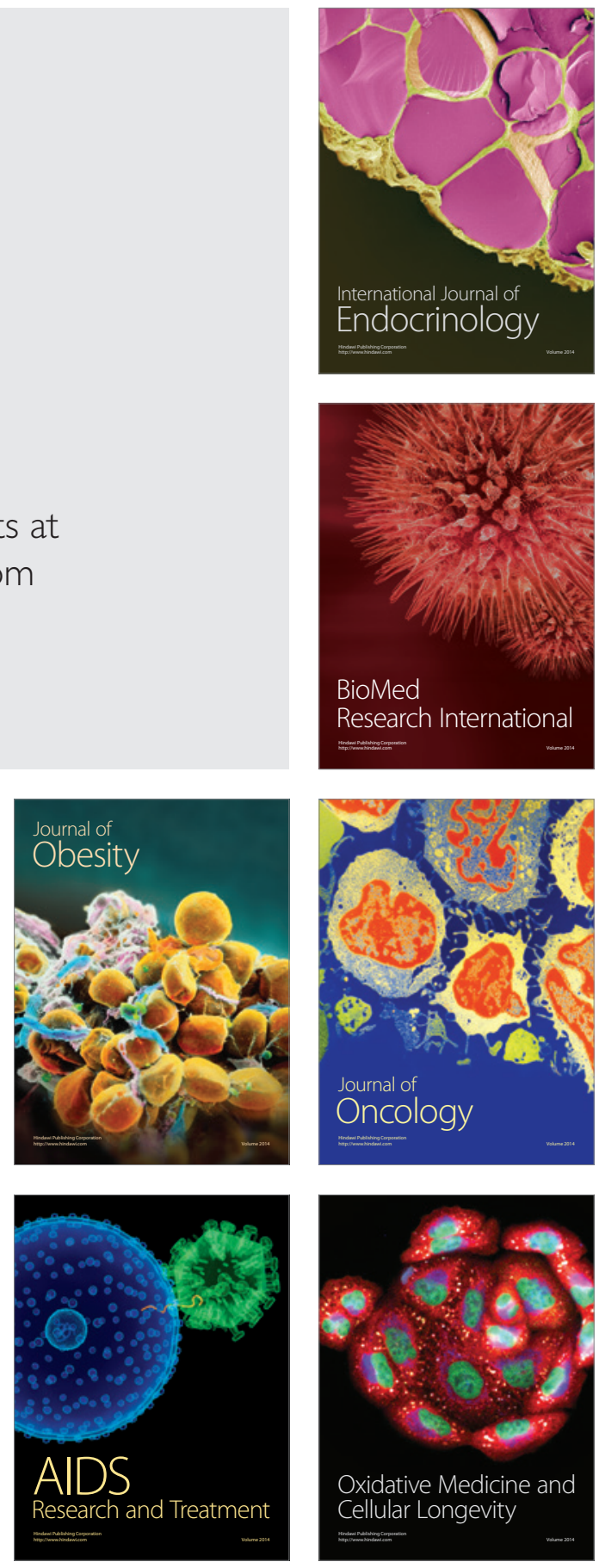